\title{
Parallaxes and proper motions of interstellar masers toward the Cygnus $\mathrm{X}$ star-forming complex
}

\author{
K. L. J. Ryg ${ }^{* a}$, A. Brunthaler ${ }^{b}$, A. Sanna ${ }^{b}$, K. M. Menten ${ }^{b}$, M. J. Reid ${ }^{c}$, H. J. van \\ Langevelde $^{d, f}$, M. Honma ${ }^{e}$, K. J. E. Torstensson ${ }^{f, d}$, and K. Fujisawa ${ }^{g}$ \\ ${ }^{a}$ Istituto di Astrofisica e Planetologia Spaziali (INAF-IAPS), Via del fosso del cavaliere 100, \\ 00133 Roma, Italy \\ ${ }^{b}$ Max-Planck-Institut für Radiostronomie (MPIfR), Auf dem Hügel 69, 53121 Bonn, Germany \\ ${ }^{c}$ Harvard Smithsonian Center for Astrophysics, 60 Garden Street, Cambridge, MA 02138, USA \\ ${ }^{d}$ Joint Institute for VLBI in Europe (JIVE), Postbus 2, 7990 AA Dwingeloo, the Netherlands \\ ${ }^{e}$ Mizusawa VLBI Observatory, National Astronomical Observatory of Japan (NAOJ), 2-21-1 \\ Osawa, Mitaka, Tokyo 181-8588, Japan \\ ${ }^{f}$ Sterrewacht Leiden, Leiden University, Postbus 9513, 2300 RA Leiden, the Netherlands \\ ${ }^{g}$ Faculty of Science, Yamaguchi University, 1677-1 Yoshida, Yamaguchi 753-8512, Japan \\ E-mail: kazi.rygl@inaf.it
}

\begin{abstract}
Whether the Cygnus X complex consists of one physically connected region of star formation or of multiple independent regions projected close together on the sky has been debated for decades. The main reason for this puzzling scenario is the lack of trustworthy distance measurements. With the measurements of trigonometric parallaxes and proper motions of five star forming regions, using $6.7 \mathrm{GHz}$ methanol masers with the European VLBI Network and $22 \mathrm{GHz}$ water masers with Very Long Baseline Array, we tried to answer this question. We found that the distances of W 75N, DR 20, DR 21, and IRAS 20290+4052 are consistent with a single distance of $1.40 \pm$ $0.08 \mathrm{kpc}$ for the Cygnus X complex. AFGL 2591, previously thought to be part of Cygnus X, was found to be located at a much greater distance of $3.33 \pm 0.11 \mathrm{kpc}$, beyond the Cygnus X region. In the future we plan to study the 3D structure of the Cygnus $\mathrm{X}$ region by improving the accuracy of our individual distances measurements through the focus on the long baselines between the EVN and the Japanese stations and the addition of another year of these EVN+Japan observations.
\end{abstract}

11 th European VLBI Network Symposium \& Users Meeting,

October 9-12, 2012

Bordeaux, France

*Speaker. 


\section{Introduction}

The Cygnus $\mathrm{X}$ region is one of the most pronounced regions of star-formation activity in the Galactic plane and stands out as a prominent source of infrared (IR) emission in our Galaxy ([13]; see e.g., the spectacular Spitzer [8] and Herschel [6] imaging). All phases of star formation and stellar evolution are observed projected across the Cygnus $\mathrm{X}$ region, including a large number of molecular clouds [21] a population of dense, massive, and dusty cores with embedded protoclusters and high-mass protostellar objects ([23], [1], [11]), ultracompact HII regions ([4], [25]), hundreds of OB-type stars (of which 65 O-type; [26] and references therein), and some supernova remnants [24].

The distance to this remarkable "mini-starburst" region has been a long-standing issue. It is still not clear whether all clouds are at the same distance or whether we see a projection of several clouds at different distances (see [21] and [24] for a detailed discussion). For example, three OB associations have photometric distance estimates between 1.2 and $1.7 \mathrm{kpc}$, a difference of more than $30 \%$. This uncertainty of $500 \mathrm{pc}$ is almost 10 times larger than the extent of the Cygnus $\mathrm{X}$ region on the sky (i.e., within a radius of about $50 \mathrm{pc}$ at a distance of $1.5 \mathrm{kpc}$ ). Kinematic distances, the most common distance method for estimating distances within the Galaxy, are not trustworthy at the Galactic longitude of Cygnus X, because the radial velocity difference between the Sun and the Cygnus $\mathrm{X}$ region is close to the typical velocity dispersion of interstellar gas in a high-mass star-forming region $\left(1-7 \mathrm{~km} \mathrm{~s}^{-1},[10]\right)$.

In this context, we used strong $6.7 \mathrm{GHz}$ methanol masers and $22 \mathrm{GHz}$ water masers as astrometric targets to measure distances of five distinct star-forming regions toward the Cygnus $\mathrm{X}$ complex. No previous parallax measurements were carried out toward this region. Using the terminology of [21], the Cygnus X complex is divided about the Cyg OB 2 cluster at $(l, b)=$ $\left(80{ }^{\circ} 22,+0.80\right)$ into a northern region, at Galactic longitudes greater than about $80^{\circ}$, and a southern region, at lower longitudes. Methanol maser emission was observed toward four star-forming regions with the European VLBI Network (EVN): W 75N, DR 20, and DR 21 in Cygnus X North, and IRAS 20290+4052 (which is likely part of the Cyg OB 2 association, see [12],[14], hereafter IRAS 20290) in Cygnus X South. The pioneering work presented in [18] already demonstrated the capability of the EVN to achieve parallax accuracies as good as $22 \mu \mathrm{m}$. Water maser emission was observed with the Very Long Baseline Array (VLBA) toward AFGL 2591, which is projected within Cygnus X South. Here, we present the work on the membership of the Cygnus X region, published in [19] and lay out our future plans to study the Cygnus X structure.

\section{Observations and data reduction}

The 6.7 GHz EVN ${ }^{1}$ observations were carried out in eight epochs between March 2009 and November 2010 under project EB039. Each observation lasted 12 hours and made use of geodeticlike observing blocks to calibrate the tropospheric zenith delays at each antenna (see [15], [2], [16] for a detailed discussion). We observed the $6.7 \mathrm{GHz}$ methanol masers toward four star-forming regions: W 75N, IRAS 20290, DR20, and DR21. Because these star-forming regions have a small

\footnotetext{
${ }^{1}$ The European VLBI Network is a joint facility of European, Chinese, South African, and other radio astronomy institutes funded by their national research councils.
} 
angular separation $(\leq 2.1$ degree) we could use the masers in $\mathrm{W} 75 \mathrm{~N}$ as a phase reference for the whole maser emission and the three background sources (angular separations $\leq 4.3$ degree). Hence, the data were phase-referenced to the $\mathrm{W} 75 \mathrm{~N}$ maser at a local standard of rest velocity of $7.1 \mathrm{~km} \mathrm{~s}^{-1}$ using a switching cycle of 1.5 minutes and the solutions were transferred to the other targets. We used the NRAO's Astronomical Image Processing System (AIPS) and ParselTongue [7] for reducing the EVN data, more details on the reduction and the parallax and proper motion analysis can be found in [18] and in [19].

The $22 \mathrm{GHz}$ water maser observations of AFGL 2591 were carried out with the VLBA ${ }^{2}$ in four epochs between November 2008 and November 2009 under program BM272H. These observations included four geodetic-like blocks for calibrating the zenith delays at each antenna. As with the EVN observations, we performed phase-referencing observations by fast-switching (every 30 seconds) between the maser and each of the four background sources. The VLBA calibration was carried out in AIPS following the procedure described in [16] (again, more observation details can be found in [19]).

\section{Membership of the Cygnus $\mathrm{X}$ region}

We obtained parallaxes and proper motions to five star-forming regions (see Table 1) and found that Cygnus X North is one physically connected complex of star-forming regions, including W 75N, DR 21, DR 20, and IRAS 20290 (and therefore probably also the Cygnus OB 2 association), located at $1.40_{-0.08}^{+0.08} \mathrm{kpc}$. This is an average of the individual distances, which range from 1.30 to $1.50 \mathrm{kpc}$. Our data are consistent with a single distance for these sources, within measurement uncertainty. Furthermore, our distance to the Cygnus X complex is similar to the photometric distance of $1.5 \mathrm{kpc}$ obtained by [5] toward the Cyg OB 2 association.

AFGL 2591, projected against the Cygnus X South region, has a much farther distance than previously assumed. This implies that (a) AFGL 2591 is not part of a single Cygnus X complex, as can be seen clearly when plotting the sources on the Galactic plane (Fig. 1) and (b) its physical properties change dramatically (discussed in [20]).

With the parallax and averaged proper motion results we calculated the 3D space velocities of the star-forming regions with respect to the Galactic center [17] (Table 1), which can, for example, be used to explore if the Cygnus X region was formed through an expanding Strömgren sphere (e.g., [9]). A 3D space velocity vector is decomposed in three space velocity components: $U$, the velocity in the direction of the Galactic center; $V$, the velocity in the direction of the Galactic rotation; and $W$, the velocity in the direction of the North Galactic Pole (NGP). In the calculation of the space velocities we used the solar peculiar motion obtained by [22] and assumed a flat Galactic rotation curve with $\theta=239 \mathrm{~km} \mathrm{~s}^{-1}$ and a solar distance to the Galactic center $R=8.3 \mathrm{kpc}$ [3]. In Fig. 2, we show not only the distance results of the Cygnus $\mathrm{X}$ star-forming regions, but we also draw their projected space motion. One can see that the space velocities of the Cygnus X star-forming regions have magnitudes between 0 up to $10 \mathrm{~km} \mathrm{~s}^{-1}$ and seem to have no particular orientation. More specifically, they do not remind of an expanding HiI region nor any other large-scale organized

\footnotetext{
${ }^{2}$ The National Radio Astronomy Observatory is a facility of the National Science Foundation operated under cooperative agreement by Associated Universities, Inc.
} 
Table 1: Parallax, proper motions, and space velocities toward Cygnus $\mathrm{X}$

\begin{tabular}{lccccccc}
\hline \hline Source & $\begin{array}{c}\text { Parallax } \\
(\mathrm{mas})\end{array}$ & $\begin{array}{c}\mathrm{D}_{\text {Sun }} \\
(\mathrm{kpc})\end{array}$ & $\begin{array}{c}\left.<\mu_{\alpha}\right\rangle \\
\left(\mathrm{mas} \mathrm{yr}^{-1}\right)\end{array}$ & $\begin{array}{c}<\mu_{\delta}> \\
\left(\mathrm{mas} \mathrm{yr}^{-1}\right)\end{array}$ & $\begin{array}{c}U \\
\left(\mathrm{~km} \mathrm{~s}^{-1}\right)\end{array}$ & $\begin{array}{c}V \\
\left(\mathrm{~km} \mathrm{~s}^{-1}\right)\end{array}$ & $\begin{array}{c}W \\
\left(\mathrm{~km} \mathrm{~s}^{-1}\right)\end{array}$ \\
\hline \hline W 75N & $0.772 \pm 0.042$ & $1.30_{-0.07}^{+0.07}$ & $-1.97 \pm 0.10$ & $-4.16 \pm 0.15$ & $-0.5 \pm 1.0$ & $3.6 \pm 2.5$ & $1.2 \pm 0.8$ \\
DR 21 & $0.666 \pm 0.035$ & $1.50_{-0.07}^{+0.08}$ & $-2.84 \pm 0.15$ & $-3.80 \pm 0.22$ & $-0.0 \pm 1.5$ & $-8.3 \pm 2.5$ & $6.6 \pm 1.3$ \\
DR 20 & $0.687 \pm 0.038$ & $1.46_{-0.08}^{+0.09}$ & $-3.29 \pm 0.13$ & $-4.83 \pm 0.26$ & $7.5 \pm 1.5$ & $-8.8 \pm 2.5$ & $5.1 \pm 1.3$ \\
IRAS 20290 & $0.737 \pm 0.062$ & $1.36_{-0.11}^{+0.12}$ & $-2.84 \pm 0.09$ & $-4.14 \pm 0.54$ & $1.9 \pm 1.8$ & $-8.2 \pm 2.5$ & $6.0 \pm 2.1$ \\
AFGL 2591 & $0.300 \pm 0.010$ & $3.33_{-0.11}^{+0.11}$ & $-1.20 \pm 0.32$ & $-4.80 \pm 0.12$ & $-12.8 \pm 3.4$ & $-10.4 \pm 2.5$ & $-22.1 \pm 4.3$ \\
\hline
\end{tabular}

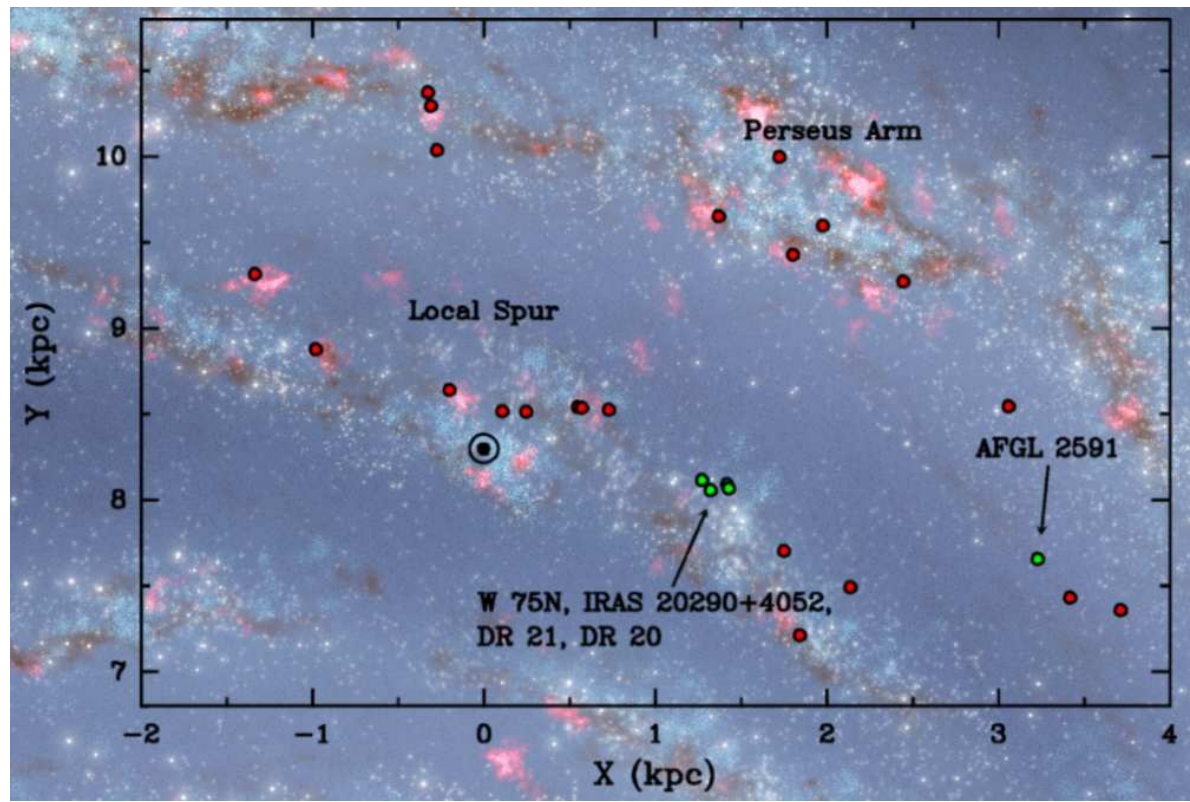

Figure 1: An artist's impression of the Galactic plane (image credit: R. Hurt, NASA/JPL-Caltech/SSC) as seen from the North Galactic Pole with the Galactic center (not shown) located at $(0,0) \mathrm{kpc}$. The Sun is located at $(0,8.3) \mathrm{kpc}$ and marked by a black circle around a black dot. Sources with known distances are marked by colored dots: green dots show the star-forming regions from this work, red dots are from the literature. The full list of literature references can be found in the caption of Fig. 11 of [19].

structure. Also, AFGL 2591 which is not part of the Cygnus X complex, clearly has very different space motions (in particular, the $W$ vector is an order of magnitude larger).

\section{Structure of the Cygnus $X$ region}

The results presented in the previous section were based on two year of EVN-only data; the calibration of the long $(9000 \mathrm{~km})$ Europe-Japan baselines proved to be more challenging since masers spots can be resolved at these baselines. While our measurements in Table 1 allowed to establish the membership of the Cygnus X region, they did not allow us to study its 3D structure, since the distance errors of the star-forming regions were overlapping. To improve our distance accuracy we conducted another four epochs of parallax observations using the EVN plus a Japanese antenna (program ER026) and will try other reduction techniques to exploit the extra angular res- 


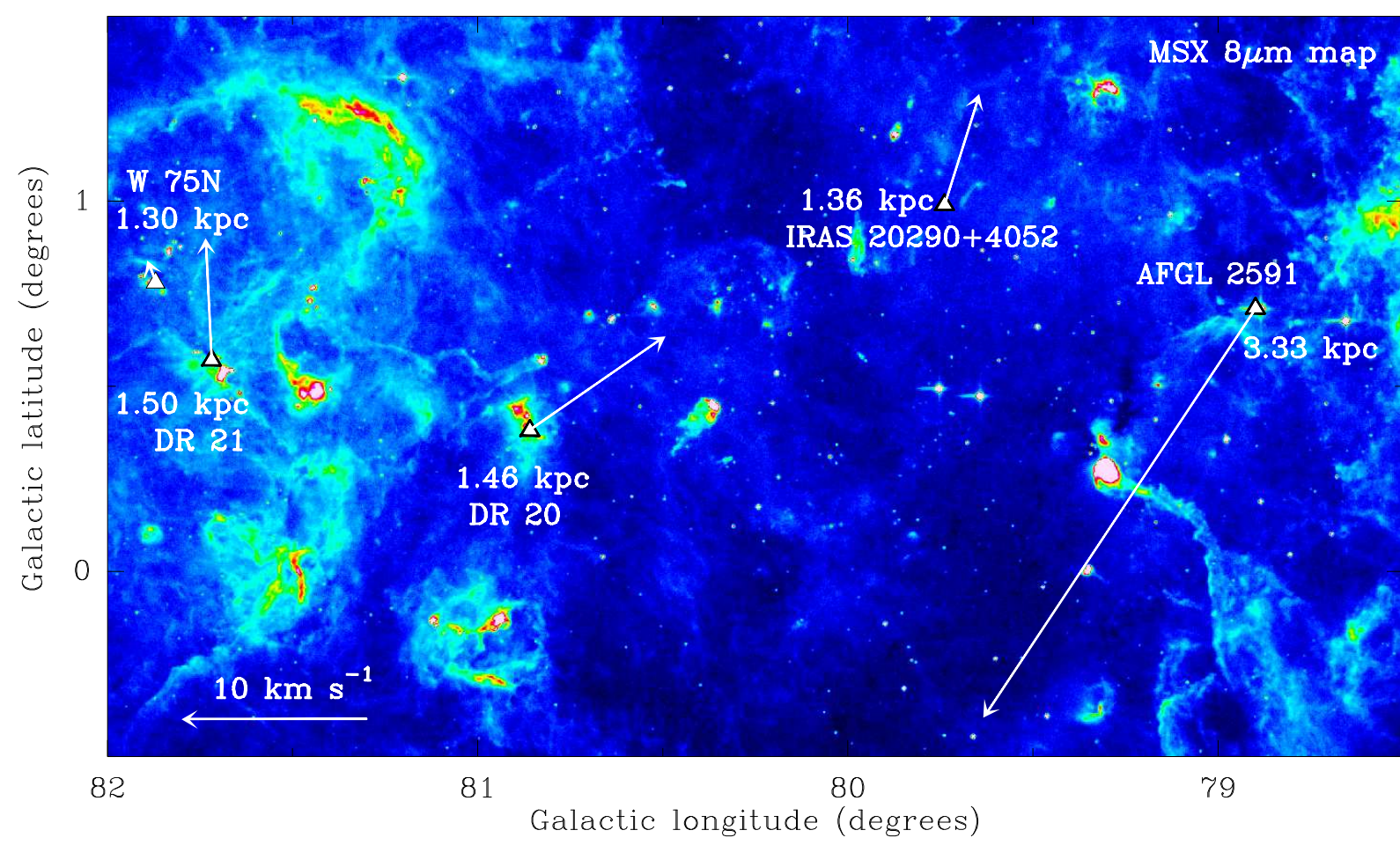

Figure 2: MSX $8 \mu \mathrm{m}$ map of the Cygnus X region overlaid with the distance results (in $\mathrm{kpc}$ ) and the resulting space motions projected on the image (arrows).

olution provided by the addition of the Europe-Japan baselines. With the increased accuracy we want to understand the relative position of the star-forming regions along the line of sight.

The Cygnus X sources covered with the EVN in this study belong mostly to Cygnus X North. For understanding more of the Cygnus $\mathrm{X}$ complex, it will be necessary to have parallaxes and proper motions toward star-forming regions in Cygnus $X$ South. For the moment, at least one source, S106 is covered by the BeSSeL survey [3] and hopefully more will come in the future.

\section{Summary}

We have presented our first astrometric study toward the Cygnus $\mathrm{X}$ region to establish if individual molecular clouds belong to the same star-forming complex, based on $6.7 \mathrm{GHz}$ methanol and $22 \mathrm{GHz}$ water maser parallaxes obtained with the EVN and the VLBA. We report the following distances: $1.30_{-0.07}^{+0.07} \mathrm{kpc}$ for W $75 \mathrm{~N}, 1.46_{-0.08}^{+0.09} \mathrm{kpc}$ for DR 20, $1.50_{-0.07}^{+0.08} \mathrm{kpc}$ for DR 21, $1.36_{-0.11}^{+0.12} \mathrm{kpc}$ for IRAS 20290+4052, and $3.33_{-0.11}^{+0.11} \mathrm{kpc}$ for AFGL 2591. While the distances of W 75N, DR 20, DR 21, and IRAS $20290+4052$ are consistent with a single distance of $1.40 \pm 0.08 \mathrm{kpc}$ for the Cygnus X complex, AFGL 2591 is located at a much greater distance than previously assumed. The space velocities of the star-forming regions in Cygnus $\mathrm{X}$ do not suggest an expanding Strömgren sphere.

\section{Acknowledgements}

KLJR thanks Radionet 3 which provided financial support to attend the 11th EVN Symposium 
and present this paper. KLJR is funded by the Agenzia Spaziale Italiana (ASI) under contract number I/005/11/0. This work was partially funded by the ERC Advanced Investigator Grant GLOSTAR (247078).

\section{References}

[1] Beuther, H., Schilke, P., Menten, K. M., et al. 2002, ApJ, 566, 945

[2] Brunthaler, A., Reid, M. J., \& Falcke, H. 2005, Future Directions in High Resolution Astronomy, 340, 455

[3] Brunthaler, A., Reid, M. J., Menten, K. M., et al. 2011, Astronomische Nachrichten, 332, 461

[4] Downes, D., \& Rinehart, R. 1966, ApJ, 144, 937

[5] Hanson, M. M. 2003, ApJ, 597, 957

[6] Hennemann, M., Motte, F., Schneider, N., et al. 2012, A\&A, 543, L3

[7] Kettenis, M., van Langevelde, H. J., Reynolds, C., et al. 2006, Astronomical Data Analysis Software and Systems XV, 351, 497

[8] Kumar, M. S. N., Davis, C. J., Grave, J. M. C., et al. 2007, MNRAS, 374, 54

[9] McCutcheon, W. H., \& Shuter, W. L. H. 1970, AJ, 75, 910

[10] Moscadelli, L., Menten, K. M., Walmsley, C. M., \& Reid, M. J. 2002, ApJ, 564, 813

[11] Motte, F., Bontemps, S., Schilke, P., et al. 2007, A\&A, 476, 1243

[12] Odenwald, S. F. 1989, AJ, 97, 801

[13] Odenwald, S. F., \& Schwartz, P. R. 1993, ApJ, 405, 706

[14] Parthasarathy, M., Jain, S. K., \& Bhatt, H. C. 1992, A\&A, 266, 202

[15] Reid, M. J., \& Brunthaler, A. 2004, ApJ, 616, 872

[16] Reid, M. J., Menten, K. M., Brunthaler, A., et al. 2009, ApJ, 693, 397

[17] Reid, M. J., Menten, K. M., Zheng, X. W., et al. 2009, ApJ, 700, 137

[18] Rygl, K. L. J., Brunthaler, A., Reid, M. J., et al. 2010, A\&A, 511, A2

[19] Rygl, K. L. J., Brunthaler, A., Sanna, A., et al. 2012, A\&A, 539, A79

[20] Sanna, A., Reid, M. J., Carrasco-González, C., et al. 2012, ApJ, 745, 191

[21] Schneider, N., Bontemps, S., Simon, et al. 2006, A\&A, 458, 855

[22] Schönrich, R., Binney, J., \& Dehnen, W. 2010, MNRAS, 403, 1829

[23] Sridharan, T. K., Beuther, H., Schilke, P., Menten, K. M., \& Wyrowski, F. 2002, ApJ, 566, 931

[24] Uyanıker, B., Fürst, E., Reich, W., et al. 2001, A\&A, 371, 675

[25] Wendker, H. J., Higgs, L. A., \& Landecker, T. L. 1991, A\&A, 241, 551

[26] Wright, N. J., Drake, J. J., Drew, J. E., \& Vink, J. S. 2010, ApJ, 713, 871 